\title{
Erratum: Symmetry-broken dissipative exchange flows in thin-film ferromagnets with in-plane anisotropy [Phys. Rev. B 96, 134434 (2017)]
}

\author{
Ezio Iacocca, T. J. Silva, and Mark A. Hoefer \\ (Received 1 December 2017; published 12 December 2017)
}

DOI: 10.1103/PhysRevB.96.219901

We have become aware of a mistake in the proportionality between the input flow and the charge current magnitude utilized in the micromagnetic simulations presented in the original paper. We have performed a new set of micromagnetic simulations for a Py nanowire of dimensions $2500 \times 100 \times 1 \mathrm{~nm}^{3}$ including both shape and magnetocrystalline anisotropy. The critical input flow estimated from the analytical calculations for this geometry presented in the original paper is $u_{c}=0.035$. The equivalent critical charge current density from spin transfer torque (STT) impinging on a $(1.2 \times 100)-\mathrm{nm}^{2}$ area located on the top left edge of the channel is found here to be $J_{c} \approx 3.54 \times 10^{11} \mathrm{~A} / \mathrm{m}^{2}$. This is almost two orders of magnitude higher than the quantity reported in the original paper. For the same STT area, dynamic solutions can be obtained for a charge current density above threshold of, e.g., $\bar{J}_{c}=3 \times 10^{12} \mathrm{~A} / \mathrm{m}^{2}$. We note that the proportionality used to estimate the critical charge current density is not exact when nonlocal dipole fields are included. In the revised figure, Fig. 1 below (to replace Fig. 5 in the original paper), the solid blue curves maintain the qualitative features of Fig. 5 in our original paper, namely, a near-homogeneous density, a decaying oscillatory fluid velocity, and a large amplitude precession.

The proportionality error reported here does not affect any of the analytical results and qualitative features of dissipative exchange flows in the presence of symmetry-breaking effects. Furthermore, the charge current density can be reduced as discussed in the original paper by increasing the area where the STT is operable. An example order of magnitude reduction in charge current density is shown in Fig. 1 by the dashed black curves.
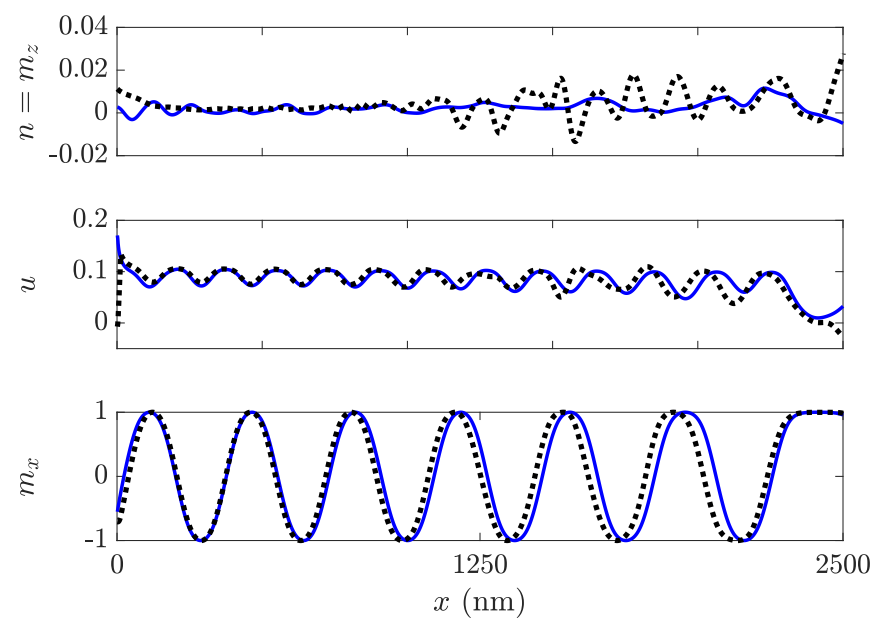

FIG. 1. Dissipative exchange flows obtained micromagnetically for a Py nanowire where magnetization precession close to the left edge is sustained by spin transfer torque from an area with dimensions $1.2 \times 100 \mathrm{~nm}^{2}$ subject to a charge current density $\bar{J}_{c}=3 \times 10^{12} \mathrm{~A} / \mathrm{m}^{2}($ the solid blue curves) and dimensions $12 \times 100 \mathrm{~nm}^{2}$ subject to a charge current density $\bar{J}_{c}=3 \times 10^{11} \mathrm{~A} / \mathrm{m}^{2}$ (the dashed black curves). The curves are snapshots of the center line along the nanowire obtained at times such that the fluid velocities partly overlap close to the left edge. 